\title{
THE SEMI-INFINITE ELASTIC STRIP*
}

\author{
BY
}

M. W. JOHNSON, Jr. (University of Wisconsin)

AND R. W. LITTLE (Oklahoma State University)

1. Introduction. We consider the two-dimensional problem of a semi-infinite elastic strip occupying the region $|y| \leq 1,0 \leq x \leq \infty$, where $x$ and $y$ are rectangular Cartesian coordinates. Let $\sigma_{x}, \sigma_{\nu}$ and $\tau$ be components of stress and $u$ and $v$ be components of displacement. We take the edges $y= \pm 1$ to be free of stress

$$
y= \pm 1, \quad 0<x<\infty: \quad \sigma_{\nu}=\tau=0 .
$$

At the edge $x=0$, one of the following conditions holds:

$$
\begin{array}{rlrl}
\sigma_{x} & =\sigma_{x b}(y), & v=v_{b}(y), \\
\tau & =\tau_{b}(y), & & u=u_{b}(y), \\
\sigma_{x} & =\sigma_{x b}(y), & & \tau=\tau_{b}(y), \\
u & =u_{b}(y), & v & =v_{b}(y)
\end{array}
$$

where the subscript $b$ indicates a given function. We also prescribe that all stress and displacement components approach zero as $x$ becomes large:

$$
\text { solution } \rightarrow 0 \text { as } x \rightarrow \infty \text {. }
$$

The equations of equilibrium and compatibility are

$$
\begin{gathered}
\frac{\partial \tau}{\partial y}+\frac{\partial \sigma_{x}}{\partial x}=0, \quad \frac{\partial \sigma_{y}}{\partial y}+\frac{\partial \tau}{\partial x}=0 \\
\left(\frac{\partial^{2}}{\partial x^{2}}+\frac{\partial^{2}}{\partial y^{2}}\right)\left(\sigma_{x}+\sigma_{v}\right)=0
\end{gathered}
$$

We will refer to problems (1.2a) and (1.2b) as the mixed problems, (1.2c) as the stress problem and (1.2d) as the displacement problem. These problems are solved in what follows by making use of an expansion in terms of four-component eigenvectors, each of which satisfies Eqs. (1.1), (1.3), (1.4) and (1.5). The satisfaction of boundary conditions (1.2) is accomplished by identifying the coefficients of the expansion with the coefficients of the expansion of the given boundary functions. The procedure here is formal. Readers are referred to [1] for a discussion of convergence of the expansion.

In addition to being of interest to plane elasticity theory, this problem has been shown by recent work on the foundations of the theory of thin plates and shells $[2,3,4]$ to be fundamental in the analysis of the boundary layer contribution to the solution of these problems.

*Received July 15, 1963: revised manuscript received April 13, 1964. The work of the first author was supported by the U. S. Army Mathematics Research Center and The Wisconsin Alumnae Research Foundation and that of the second author by a loan from the Ford Foundation. Part of the material presented in this paper is from the Ph.D. thesis of the second author. 
2. Formulation of boundary value problem. The usual procedure is to derive from the system (1.4) and (1.5) a single fourth order differential equation for Airy's stress function. We will find it convenient instead to work with an equivalent system of four first order equations. This idea is, in fact, the heart of the method of solution presented here.

We introduce a new function $p(x, y)$ satisfying

$$
\frac{\partial \sigma_{x}}{\partial y}-\frac{\partial p}{\partial x}=0 .
$$

Using Eqs. (1.4) and (2.1), we may write the compatibility equation (1.5) in the following form:

$$
\frac{\partial^{2}}{\partial x^{2}}\left(2 \sigma_{x}+\sigma_{y}\right)+\frac{\partial^{2} p}{\partial x \partial y}=0 .
$$

Equation (2.2) can be integrated with respect to $x$ to yield

$$
\frac{\partial p}{\partial y}+\frac{\partial}{\partial x}\left(2 \sigma_{x}+\sigma_{y}\right)=0,
$$

where the function of integration is set to zero to satisfy condition (1.3).

The introduction of the variable $p$ and the derivation of the compatibility equation in the form (2.3) allow us to write the system (1.4), (2.1) and (2.3) in the vector form

where

$$
\frac{\partial \mathrm{f}}{\partial y}+U \cdot \frac{\partial \mathrm{f}}{\partial x}=0
$$

$$
\mathbf{f}=\left(\begin{array}{r}
\sigma_{\nu} \\
-\tau \\
\sigma_{x} \\
p
\end{array}\right), \quad U=\left(\begin{array}{rrrr}
0 & -1 & 0 & 0 \\
0 & 0 & -1 & 0 \\
0 & 0 & 0 & -1 \\
1 & 0 & 2 & 0
\end{array}\right) .
$$

The form (2.4) of the basic equation is motivated by the fact that the ordinary differential equation obtained upon using the separation of variables procedure will be in a standard vector form.

We next examine the boundary conditions on vector $f$. Two of the stress-strain relations are:

$$
\begin{aligned}
& \frac{\partial u}{\partial x}=\frac{1}{E}\left(\sigma_{x}-\nu \sigma_{y}\right), \\
& \frac{\partial v}{\partial y}=\frac{1}{E}\left(\sigma_{\nu}-v \sigma_{x}\right),
\end{aligned}
$$

where $\nu$ is Poisson's ratio and $E$ is Young's modulus. From Eqs. (1.4), (2.1) and (2.7) we obtain

$$
\frac{\partial^{2} u}{\partial x \partial y}=\frac{1}{E}\left(\frac{\partial p}{\partial x}+\nu \frac{\partial \tau}{\partial x}\right) .
$$

Integration of Eq. (2.9) and use of Eq. (1.3) yields 


$$
\frac{\partial u}{\partial y}=\frac{1}{E}(p+\nu \tau)
$$

Using Eqs. (2.8) and (2.10) we see that the boundary conditions (1.2) are equivalent, respectively, to specification on edge $x=0$ of

$$
\begin{gathered}
f^{(1)}=\sigma_{\nu b}=\nu \sigma_{x b}+E \frac{d v_{b}}{d y}, \quad f^{(3)}=\sigma_{x b}, \\
f^{(2)}=-\tau_{b}, \quad f^{(4)}=p_{b}=-\nu \tau_{b}+E \frac{d u_{b}}{d y}, \\
f^{(2)}=-\tau_{b}, \quad f^{(3)}=\sigma_{x b}, \\
f^{(1)}-\nu f^{(3)}=\sigma_{\nu b}-\nu \sigma_{x b}=E \frac{d v_{b}}{d y}, \\
f^{(4)}-\nu f^{(2)}=p_{b}+\nu \tau_{b}=E \frac{d u_{b}}{d y},
\end{gathered}
$$

Conditions (1.1) and (1.3) take the form

$$
\begin{aligned}
& x \rightarrow \infty, \quad|y|<1: \quad \mathrm{f} \rightarrow 0 \\
& 0<x<\infty, \quad y= \pm 1: \quad f^{(1)}=f^{(2)}=0
\end{aligned}
$$

We seek a representation of the solution $f$ in the interior of the strip in terms of its value $f_{b}$ on the end. We will then show how to proceed from this representation to the solution of problems with end conditions (1.2).

3. Series solution. We take the solution in the form of an infinite series

$$
\mathbf{f}=\sum_{n} c_{n} Z_{n}(y) e^{i \alpha_{n} x},
$$

where $c_{n}$ and $\alpha_{n}$ are constants to be determined as are the vector functions $Z_{n}$. Substitution of the series (3.1) into the differential Eq. (2.4) yields the following system of ordinary differential equations for the functions $\mathbf{Z}_{n}$ :

$$
\mathbf{Z}_{n}^{\prime}+i \alpha_{n} U \cdot \mathbf{Z}_{n}=0,
$$

where the prime denotes differentiation with respect to $y$. From conditions (2.13) we obtain the following boundary conditions on the functions $Z_{n}$ :

$$
y= \pm 1: \quad Z_{n}^{(1)}=Z_{n}^{(2)}=0
$$

System (3.2) with the boundary conditions (3.3) forms an eigenvalue problem. Its solution is elementary as the system (3.2) has constant coefficients. The eigenvectors divide themselves into two sets, those where the first component $Z_{n}^{(1)}$ is odd in $y$, and those where it is even.

The odd eigenvectors are given by

$$
\begin{aligned}
& { }_{0} Z_{n}^{(1)}=-{ }_{0} \alpha_{n}^{2}\left[{ }_{0} \alpha_{n} y \sinh { }_{0} \alpha_{n} \cosh { }_{0} \alpha_{n} y-{ }_{0} \alpha_{n} \cosh { }_{0} \alpha_{n} \sinh { }_{0} \alpha_{n} y\right], \\
& { }_{0} Z_{n}^{(2)}=i_{0} \alpha_{n}^{2}\left[{ }_{0} \alpha_{n} y \sinh { }_{0} \alpha_{n} \sinh { }_{0} \alpha_{n} y-\left({ }_{0} \alpha_{n} \cosh { }_{0} \alpha_{n}-\sinh { }_{0} \alpha_{n}\right) \cosh { }_{0} \alpha_{n} y\right], \\
& { }_{0} Z_{n}^{(3)}={ }_{0} \alpha_{n}^{2}\left[{ }_{0} \alpha_{n} y \sinh { }_{0} \alpha_{n} \cosh { }_{0} \alpha_{n} y-\left({ }_{0} \alpha_{n} \cosh { }_{0} \alpha_{n}-2 \sinh { }_{0} \alpha_{n}\right) \sinh { }_{0} \alpha_{n} y\right], \\
& { }_{0} Z_{n}^{(4)}=-i_{0} \alpha_{n}^{2}\left[{ }_{0} \alpha_{n} y \sinh { }_{0} \alpha_{n} \sinh { }_{0} \alpha_{n} y-\left({ }_{0} \alpha_{n} \cosh { }_{0} \alpha_{n}-3 \sinh { }_{0} \alpha_{n}\right) \cosh { }_{0} \alpha_{n} y\right],
\end{aligned}
$$


while the even eigenvectors are given by

$$
\begin{aligned}
& { }_{0} Z_{n}^{(1)}=-{ }_{e} \alpha_{n}^{2}\left[{ }_{e} \alpha_{n} y \cosh { }_{e} \alpha_{n} \sinh { }_{e} \alpha_{n} y-{ }_{e} \alpha_{n} \sinh { }_{e} \alpha_{n} \cosh { }_{e} \alpha_{n} y\right] \text {, } \\
& { }_{e} Z_{n}^{(2)}=i_{e} \alpha_{n}^{2}\left[\alpha_{e} \alpha_{n} y \cosh { }_{e} \alpha_{n} \cosh { }_{e} \alpha_{n} y-\left({ }_{e} \alpha_{n} \sinh { }_{e} \alpha_{n}-\cosh { }_{e} \alpha_{n}\right) \sinh { }_{e} \alpha_{n} y\right] \text {, } \\
& . Z_{n}^{(3)}={ }_{e} \alpha_{n}^{2}\left[{ }_{e} \alpha_{n} y \cosh { }_{e} \alpha_{n} \sinh { }_{e} \alpha_{n} y-\left({ }_{e} \alpha_{n} \sinh { }_{e} \alpha_{n}-2 \cosh { }_{e} \alpha_{n}\right) \cosh { }_{e} \alpha_{n} y\right] \text {, } \\
& { }_{\odot} Z_{n}^{(4)}=-i_{e} \alpha_{n}^{2}\left[\alpha_{e} \alpha_{n} y \cosh { }_{\odot} \alpha_{n} \cosh { }_{\odot} \alpha_{n} y-\left({ }_{\odot} \alpha_{n} \sinh { }_{\odot} \alpha_{n}-3 \cosh { }_{e} \alpha_{n}\right) \sinh { }_{\odot} \alpha_{n} y\right] \text {. }
\end{aligned}
$$

The eigenvalues ${ }_{0} \alpha_{n}$ and ${ }_{\odot} \alpha_{n}$ are solutions of the following transcendental equations:

$$
\sinh \left(2_{0} \alpha_{n}\right)=2_{0} \alpha_{n}, \quad \sinh \left(2_{e} \alpha_{n}\right)=-2_{e} \alpha_{n} .
$$

Equations (3.6) have been investigated ${ }^{1}$ by Hillman and Salzer [10] and by Robbins and Smith [11]. These equations have infinite roots in each quadrant of the complex $\alpha$-plane. To make the solution (3.1) satisfy condition (2.12), we use only eigenfunctions in (3.1) associated with roots $\alpha_{n}$ in the upper half-plane. Hence, the sum (3.1) is extended over all eigenvectors (3.4) and (3.5) whose eigenvalues have positive imaginary parts. We note that these occur in pairs in the first and second quadrants such that, if $\alpha_{n}$ is a root, $\mathrm{so}^{2}$ is $-\alpha_{n}^{*}$.

It remains to discuss the satisfaction of the boundary conditions along the edge $x=0$. Letting $x$ be zero in Eq. (3.1) yields

$$
\sum_{n} c_{n} Z_{n}(y)=\mathbf{f}_{b}
$$

where $f_{b}$ is the vector of end values of $f$. From Eq. (3.7) we can determine the constants $c_{n}$ in terms of end values $f_{b}$. Note that the vectors $Z_{n}$ do not satisfy the condition of orthogonality, ${ }^{3}$

$$
\int_{-1}^{+1} Z_{k}^{\dagger} \cdot U \cdot Z_{n} d y=0, \quad k \neq n
$$

However, there exists a set of vectors $\mathbf{W}_{n}$ which are biorthogonal to $Z_{n}$ and satisfy the condition

$$
\int_{-1}^{+1} \mathrm{~W}_{k}^{\dagger} \cdot U \cdot Z_{n} d y=0, \quad k \neq n
$$

and these are obtained in Section 4. Proceeding formally, we premultiply both sides of Eq. (3.7) by $\mathrm{W}_{n}^{\dagger} \cdot U$, integrate and use Eq. (3.9) to obtain

$$
c_{n}=K_{n}^{-1} \int_{-1}^{+1} \mathrm{~W}_{n}^{\dagger} \cdot U \cdot \mathbf{f}_{b} d y, \quad K_{n}=\int_{-1}^{+1} \mathrm{~W}_{n}^{\dagger} \cdot U \cdot Z_{n} d y
$$

In expanded form, Eq. (3.10) gives

$$
\begin{aligned}
& c_{n}=K_{n}^{-1} \int_{-1}^{+1}\left[W_{n}^{*(4)} f_{b}^{(1)}-W_{n}^{*(1)} f_{b}^{(2)}+\left(2 W_{n}^{*(4)}-W_{n}^{*(2)}\right) f_{b}^{(3)}-W_{n}^{*(3)} f_{b}^{(4)}\right] d y, \\
& K_{n}=\int_{-1}^{+1}\left[W_{n}^{*(4)} Z_{n}^{(1)}-W_{n}^{*(1)} Z_{n}^{(2)}+\left(2 W_{n}^{*(4)}-W_{n}^{*(2)}\right) Z_{n}^{(3)}-W_{n}^{*(3)} Z_{n}^{(4)}\right] d y .
\end{aligned}
$$

${ }^{1}$ To obtain the equations considered by these writers we substitute $s_{n}=i \alpha_{n}^{*}$, where the ( )* denotes the complex conjugate. Equations (3.6) become $\sin \left(2_{0} s_{n}\right)=2_{0} s_{n}$ and $\sin \left(2_{e} s_{n}\right)=-2_{e} s_{n}$ respectively. 2( )* denotes the complex conjugate.

${ }^{3}$ A superscript $T$ indicates the transpose of a vector or matrix. ( $) \dagger$ denotes the conjugate transpose. 
With $c_{n}$ given by Eqs. (3.11), Eq. (3.1) gives the desired representation of $f$ in terms of its boundary values $f_{b}$. In section 5 we show how to proceed from this representation to the satisfactoin of boundary conditions of the form (1.2). Note that the differential equations and the boundary conditions (1.1) and (1.3) are satisfied term by term by the series (3.1).

4. The biorthogonal vectors $W_{m}$. The vectors $W_{m}$ satisfy the adjoint equation

$$
\mathrm{W}_{n}^{\prime}+i \alpha_{n}^{*} U^{\dagger} \cdot \mathrm{W}_{n}=0 \text {. }
$$

Boundary conditions on $W_{n}$ are to be chosen so that the following equation is satisfied:

$$
\int_{-1}^{+1}\left(\mathrm{~W}_{k}^{\dagger} \cdot \mathbf{Z}_{j}^{\prime}+\mathrm{W}_{k}^{+\prime} \cdot \mathbf{Z}_{j}\right) d y=\int_{-1}^{+1}\left(\mathrm{~W}_{k}^{\dagger} \cdot Z_{i}\right)^{\prime} d y=\left[\mathrm{W}_{k}^{\dagger} \cdot \mathbf{Z}_{i}\right]_{-1}^{+1}=0 .
$$

Since $Z_{n}$ satisfies the conditions (3.3), we use the following boundary conditions for $\mathrm{W}_{n}$ in order that Eq. (4.2) will hold:

$$
y= \pm 1: \quad W_{n}^{(3)}=W_{n}^{(4)}=0 .
$$

Substituting into Eq. (4.2) from Eqs. (3.2) and (4.1), we obtain

$$
\int_{-1}^{+1}\left[\mathrm{~W}_{k}^{\dagger} \cdot\left(-i \alpha_{j} U \cdot Z_{j}\right)+\left(i \alpha_{k}\left(U^{\dagger} \cdot \mathrm{W}_{k}\right)^{\dagger}\right) \cdot Z_{i}\right] d y=0,
$$

or

$$
\left(\alpha_{k}-\alpha_{j}\right) \int_{-1}^{+1} \mathrm{~W}_{k}^{\dagger} \cdot U \cdot Z_{j} d y=0,
$$

which yields the biorthogonality condition (3.9).

The eigenvectors $\mathrm{W}_{n}$ are obtained by solving the eigenvalue problem given by Eqs. (4.1) and (4.3). The eigenvectors again split into two sets. Corresponding to the eigenvalues given by Eq. (3.6a) we obtain:

$$
\begin{aligned}
& { }_{0} W_{n}^{(1)}=-i\left[{ }_{0} \alpha_{n}^{*} y \sinh { }_{0} \alpha_{n}^{*} \sinh { }_{0} \alpha_{n}^{*} y-\left({ }_{0} \alpha_{n}^{*} \cosh { }_{0} \alpha_{n}^{*}+\sinh { }_{0} \alpha_{n}^{*}\right) \cosh { }_{0} \alpha_{n}^{*} y\right], \\
& { }_{0} W_{n}^{(2)}=\left[{ }_{0} \alpha_{n}^{*} y \sinh { }_{0} \alpha_{n}^{*} \cosh { }_{0} \alpha_{n}^{*} y-\left({ }_{0} \alpha_{n}^{*} \cosh { }_{0} \alpha_{n}^{*}+2 \sinh { }_{0} \alpha_{n}^{*}\right) \sinh { }_{0} \alpha_{n}^{*} y\right], \\
& { }_{0} W_{n}^{(3)}=-i\left[{ }_{0} \alpha_{n}^{*} y \sinh { }_{0} \alpha_{n}^{*} \sinh { }_{0} \alpha_{n}^{*} y-\left({ }_{0} \alpha_{n}^{*} \cosh { }_{0} \alpha_{n}^{*}-\sinh { }_{0} \alpha_{n}^{*}\right) \cosh { }_{0} \alpha_{n}^{*} y\right], \\
& { }_{0} W_{n}^{(4)}=\left[{ }_{0} \alpha_{n}^{*} y \sinh { }_{0} \alpha_{n}^{*} \cosh { }_{0} \alpha_{n}^{*} y-{ }_{0} \alpha_{n}^{*} \cosh { }_{0} \alpha_{n}^{*} \sinh { }_{0} \alpha_{n}^{*} y\right] .
\end{aligned}
$$

The eigenvectors which correspond to the eigenvalues given by Eq. (3.6b) are

$$
\begin{aligned}
& . W_{n}^{(1)}=-i\left[{ }_{e} \alpha_{n}^{*} y \cosh { }_{e} \alpha_{n}^{*} \cosh { }_{e} \alpha_{n}^{*} y-\left({ }_{e} \alpha_{n}^{*} \sinh { }_{e} \alpha_{n}^{*}+\cosh { }_{e} \alpha_{n}^{*}\right) \sinh { }_{e} \alpha_{n}^{*} y\right] \text {, } \\
& \text {. } W_{n}^{(2)}=\left[{ }_{e} \alpha_{n}^{*} y \cosh { }_{e} \alpha_{n}^{*} \sinh { }_{e} \alpha_{n}^{*} y-\left({ }_{e} \alpha_{n}^{*} \sinh { }_{\odot} \alpha_{n}^{*}+2 \cosh { }_{e} \alpha_{n}^{*}\right) \cosh { }_{\odot} \alpha_{n}^{*} y\right] \text {, } \\
& { }_{\odot} W_{n}^{(3)}=-i\left[{ }_{\odot} \alpha_{n}^{*} y \cosh { }_{e} \alpha_{n}^{*} \cosh { }_{\odot} \alpha_{n}^{*} y-\left({ }_{e} \alpha_{n}^{*} \sinh { }_{\odot} \alpha_{n}^{*}-\cosh { }_{\odot} \alpha_{n}^{*}\right) \sinh { }_{e} \alpha_{n}^{*} y\right] \text {, } \\
& . W_{n}^{(4)}=\left[{ }_{e} \alpha_{n}^{*} y \cosh { }_{e} \alpha_{n}^{*} \sinh { }_{\odot} \alpha_{n}^{*} y-{ }_{e} \alpha_{n}^{*} \sinh { }_{e} \alpha_{n}^{*} \cosh { }_{e} \alpha_{n}^{*} y\right] \text {. }
\end{aligned}
$$

Substitution from Eqs. (3.4) and (4.6) into Eq. (3.11) and integration yields

$$
{ }_{0} K_{n}=8\left({ }_{0} \alpha_{n}\right)^{2} \sinh ^{4}{ }_{0} \alpha_{n} \text {. }
$$

Similarly, Eqs. (3.5) and (4.7) yield

$$
{ }_{\odot} K_{n}=8\left({ }_{e} \alpha_{n}\right)^{2} \cosh ^{4}{ }_{e} \alpha_{n} \text {. }
$$


5. Boundary conditions on edge $x=0$. We wish to show how the constants $c_{n}$ are determined in terms of the given functions of Eqs. (2.11). For the mixed problems, (2.11a) and (2.11b), this determination can be done in an explicit manner. For problem (2.11a), we set

$$
f_{b}^{(1)}=\sigma_{y b}, \quad f_{b}^{(2)}=\sum c_{n} Z_{n}^{(2)}(y), \quad f_{b}^{(3)}=\sigma_{x b}, \quad f_{b}^{(4)}=\sum c_{n} Z_{n}^{(4)}(y) .
$$

Substitution of Eq's. (5.1) into Eq. (3.11) for $c_{n}$ yields

$$
\begin{aligned}
c_{n}=K_{n}^{-1} \int_{-1}^{+1}\left[W_{n}^{*(4)} \sigma_{y b}+\left(2 W_{n}^{*(4)}\right.\right. & \left.\left.-W_{n}^{*(2)}\right) \sigma_{x b}\right] d y \\
& -K_{n}^{-1} \sum_{i} c_{j} \int_{-1}^{+1}\left[W_{n}^{*(1)} Z_{j}^{(2)}+W_{n}^{*(3)} Z_{i}^{(4)}\right] d y .
\end{aligned}
$$

The functions $\mathrm{Z}_{n}^{(2)}$ and $Z_{n}^{(4)}$ form the components of a two-dimensional vector whose biorthogonal vector has components $W_{n}^{(1)}$ and $W_{n}^{(3)}$. Substitution from Eqs. (3.4), (3.5), (4.6) and (4.7) shows that

$$
\int_{-1}^{+1}\left[W_{n}^{*(1)} Z_{i}^{(2)}+W_{n}^{*(3)} Z_{j}^{(4)}\right] d y= \begin{cases}0, & n \neq j, \\ -\frac{1}{2} K_{n}, & n=j .\end{cases}
$$

Utilizing Eq. (5.3), we can rewrite Eq. (5.2) in the form

$$
c_{n}=2 K_{n}^{-1} \int_{-1}^{+1}\left[W_{n}^{*(4)} \sigma_{y b}+\left(2 W_{n}^{*(4)}-W_{n}^{*(2)}\right) \sigma_{x b}\right] d y,
$$

which gives the constants $c_{n}$ directly from the given boundary functions $\sigma_{y b}$ and $\sigma_{x b}$.

The other mixed problem (2.11b) is solved in a similar manner and also involves an inner biorthogonality. The result is

$$
c_{n}=2 K_{n}^{-1} \int_{-1}^{+1}\left[W_{n}^{*(1)} \tau_{b}-W_{n}^{*(3)} p_{b}\right] d y .
$$

The stress and displacement problems do not exhibit an inner biorthogonality property and, therefore, do not reduce to a form from which the coefficients $c_{n}$ may be directly obtained. For problem (2.11c) we obtain

$$
\begin{array}{ll}
f_{b}^{(1)}=\sum c_{n} Z_{n}^{(1)}(y), & f_{b}^{(2)}=-\tau_{b}, \\
f_{b}^{(3)}=\sigma_{x b}, & f_{b}^{(4)}=\sum c_{n} Z_{n}^{(4)}(y) .
\end{array}
$$

Substitution into Eq. (3.11) yields

$$
c_{n}=K_{n}^{-1} \sum_{j} c_{i} \int_{-1}^{+1}\left[W_{n}^{*(4)} Z_{i}^{(1)}-W_{n}^{*(3)} Z_{i}^{(4)}\right] d y+F_{n},
$$

where

$$
F_{n}=K_{n}^{-1} \int_{-1}^{+1}\left[W_{n}^{*(1)} \tau_{b}+\left(2 W_{n}^{*(4)}-W_{n}^{*(2)}\right) \sigma_{x b}\right] d y .
$$

Making use of Eqs. (3.4), (3.5) and (4.6) through (4.9), we may carry out the integration in (5.7) to obtain

$$
c_{n}=\sum_{i} R_{n j} c_{i}+2 F_{n}, \quad n=1,2,3, \cdots,
$$


where

$$
R_{n j}=\left\{\begin{array}{lll}
\frac{\left.-4{ }_{0} \alpha_{j}^{3}{ }_{0} \alpha_{n}^{2} \sinh ^{2}{ }_{0} \alpha_{j}-{ }_{0} \alpha_{j}^{2} \sinh ^{2}{ }_{0} \alpha_{n}\right)}{\left.\left({ }_{0} \alpha_{j}+{ }_{0} \alpha_{n}\right){ }_{0} \alpha_{i}^{2}-{ }_{0} \alpha_{n}^{2}\right)^{2} \sinh ^{4}{ }_{0} \alpha_{n}}, & n \neq j, & n \text { and } j \text { both odd }, \\
\frac{4{ }_{e} \alpha_{j}^{3}\left({ }_{e} \alpha_{n}^{2} \cosh ^{2}{ }_{e} \alpha_{j}-{ }_{e} \alpha_{i}^{2} \cosh ^{2}{ }_{e} \alpha_{n}\right)}{\left({ }_{e} \alpha_{j}+{ }_{e} \alpha_{n}\right)\left({ }_{e} \alpha_{i}^{2}-{ }_{e} \alpha_{n}^{2}\right)^{2} \cosh ^{4}{ }_{e} \alpha_{n}}, & n \neq j, & n \text { and } j \text { both even, } \\
0, \quad n=j \text { or } n \neq j \text { with } n \text { odd }, j \text { even or } n \text { even, } j \text { odd. }
\end{array}\right.
$$

Equation (5.9) is a system of infinite equations in infinitely many unknowns, which can be solved by truncation to obtain values of $c_{n}$ to any desired degree of accuracy.

For the displacement problem, (2.11d), we write

$$
\begin{aligned}
f_{b}^{(1)} & =E v_{b}^{\prime}+\nu \sum_{n} c_{n} Z_{n}^{(3)}, & f_{b}^{(2)} & =\sum_{n} c_{n} Z_{n}^{(2)}, \\
f_{b}^{(3)} & =\sum_{n} c_{n} Z_{n}^{(3)}, & f_{b}^{(4)} & =E u_{b}^{\prime}+\nu \sum_{n} c_{n} Z_{n}^{(2)},
\end{aligned}
$$

which leads to a system of equations for the $c_{i}$ 's of the same form as (5.9).

6. Convergence of series expansions. For the series expansions to converge to the given boundary functions, it is necessary that the boundary functions be self-equilibrating:

$$
\int_{-1}^{+1} f_{b}^{(2)} d s=\int_{-1}^{+1} f_{b}^{(3)} d s=\int_{-1}^{+1} f_{b}^{(3)} s d s=\int_{-1}^{+1}\left(s^{2}-1\right) f_{b}^{(4)} d s=0 .
$$

The meaning of restrictions (6.1) is made clear by showing that they must be satisfied by any solution of Eqs. (1.1), (1.3), (1.4) and (1.5) for $0<x<\infty$ and as $x \rightarrow 0$. Static equilibrium considerations ${ }^{1}$ show that the net force and moment on any section vanish for all $x$,

$$
\int_{-1}^{+1} \sigma_{x} d y=\int_{-1}^{+1} \sigma_{x} y d y=\int_{-1}^{+1} \tau d y=0, \quad 0<x<\infty,
$$

which are just the first three of conditions (6.1). Integration by parts gives

$$
\frac{\partial}{\partial x} \int_{-1}^{+1}\left(y^{2}-1\right) p d y=\int_{-1}^{+1}\left(y^{2}-1\right) \frac{\partial \sigma_{x}}{\partial y} d y=-2 \int_{-1}^{+1} y \sigma_{x} d y
$$

which vanishes by (6.2). Hence,

$$
\int_{-1}^{+1}\left(y^{2}-1\right) p d y=\text { constant }=0
$$

for all $x>0$ if we use (1.3). Condition (6.4) agrees with condition (6.1) •

Conditions in addition to (6.1) that are sufficient to ensure uniform convergence of the series to the given boundary values are discussed in [1]. We here examine numerically the convergence for a particular example of the stress problem. Consider the boundary stresses

$$
\tau_{b}=-\sin \pi y, \quad \sigma_{x b}=-3 y+10 y^{3}-7 y^{5},
$$

which satisfy the first three conditions of (6.1). A CDC 1604 computer was programmed to solve for the vector $F_{n}$ from (5.8) and the matrix $R_{n i}$ from (5.10). The linear system

${ }^{1}$ This result can also be obtained from consideration of the basic equations (1.1), (1.3), (1.4) and (1.5). 


\section{TABLE I}

First quadrant eigenvalues from [10] and [11].

\begin{tabular}{|c|c|c|c|c|}
\hline$n$ & $\operatorname{Re}{ }_{0} \alpha_{n}$ & $\operatorname{Im}{ }_{0} \alpha_{n}$ & $\operatorname{Re}{ }_{e} \alpha_{n}$ & $\operatorname{Im}{ }_{0} \alpha_{n}$ \\
\hline 1 & 1.384339 & 3.748838 & 1.125365 & 2.106196 \\
\hline 2 & 1.676105 & 6.949980 & 1.551575 & 5.356269 \\
\hline 3 & 1.858384 & 10.119259 & 1.775544 & 8.536683 \\
\hline 4 & 1.991571 & 13.277274 & 1.929405 & 11.699178 \\
\hline 5 & 2.096626 & 16.429872 & 2.046853 & 14.854060 \\
\hline 6 & 2.183398 & 19.579409 & 2.141891 & 18.004933 \\
\hline 7 & 2.257320 & 22.727036 & 2.221723 & 21.153414 \\
\hline 8 & 2.321714 & 25.873384 & 2.290553 & 24.300342 \\
\hline 9 & 2.378758 & 29.018831 & 2.351048 & 27.446203 \\
\hline 10 & 2.429959 & 32.163617 & 2.405013 & 30.591295 \\
\hline
\end{tabular}

Asymptotic formulae are as follows: $\operatorname{Re}{ }_{0} \alpha_{n} \sim .5 \log _{e}(4 n+1) \pi$,

$\operatorname{Im}_{n} \alpha_{0} \sim(n+.25) \pi, \operatorname{Re}{ }_{e} \alpha_{n} \sim .5 \log _{e}(4 n+3)_{\pi}$,

$\operatorname{Im}{ }_{{ }_{0} \alpha_{n}} \sim(n+.75) \pi$

(5.9) was then solved and the coefficients $c_{n}$ were used to check the values of $\tau$ and $\sigma_{z}$ on the boundary $x=0$ given by the series against the specified functions (6.5). The eigenvalues are given in Table 1. The results for five and ten sets of paired eigenvalues (in the first and second quadrants) are shown in Figures 1 and 2 and show a rapid rate of convergence. Note from (5.10) that Eqs. (5.9) uncouple into two sets, one set associated with odd eigenvalues and one set with even. The authors found the calculations simple to make because the program could be written in complex arithmetic.

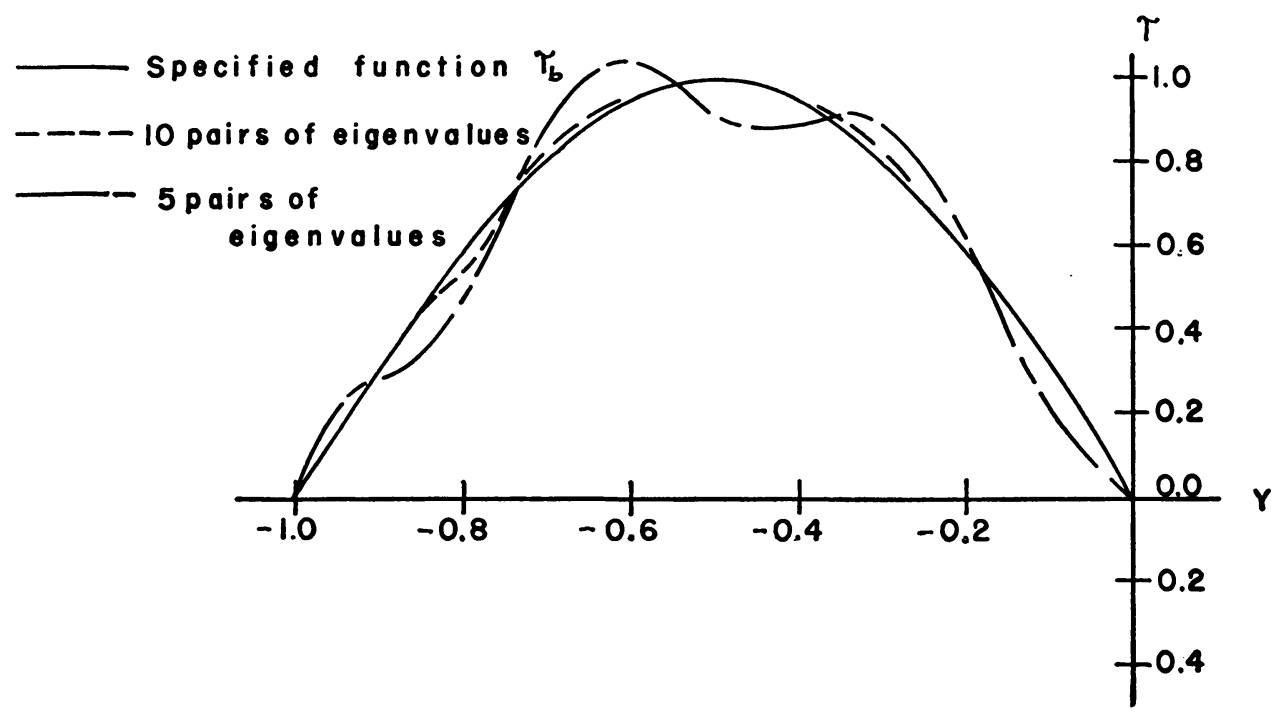

FIG. 1. 


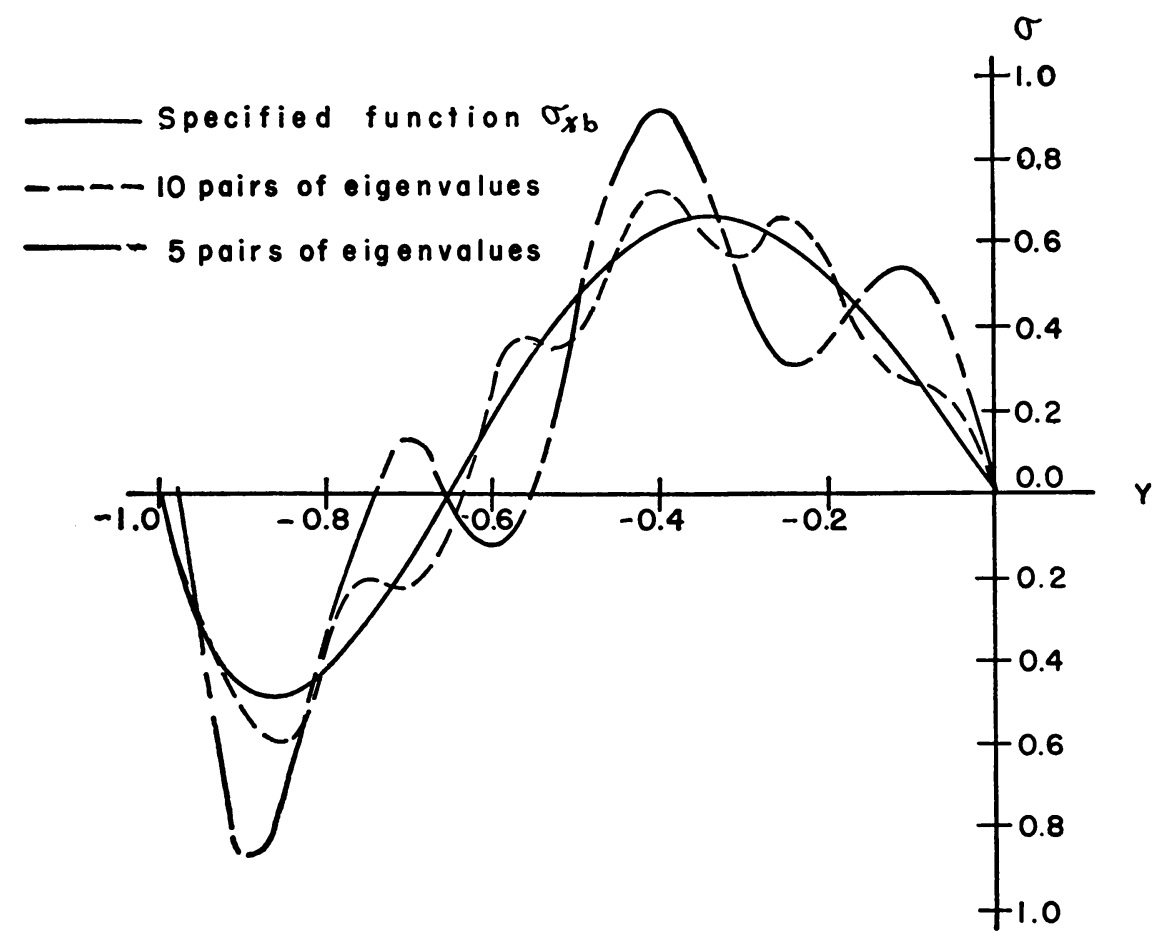

Fra. 2.

7. Concluding remarks. Smith [5] considered the problem of the bending of a semi-infinite strip loaded by moments along the short edge, his problem being mathematically equivalent to our mixed problems. Smith obtains a solution of his problem directly in a two-component vector form. Eqs. (5.4) and (5.5) agree with the corresponding results of Smith if the eigenvalues are carefully compared ${ }^{1}$. We note that Smith's form of the solution can also be obtained, for the mixed problems, by use of the Fourier integral [8]. The mixed problems have also been considered by Horvay [6] and by Horvay and Born [7] by another method. Their method is extended to the stress problem, but we believe that the method given here provides a more simple ${ }^{2}$ and accurate numerical method. At the same time, it answers the question of why the stress and displacement problems are so much harder to solve than the mixed problems.

Note added in revision. Since this paper was first submitted a paper has appeared [12] which treats the stress and displacement problems by means of the Laplace transform and the calculus of residues. The resulting series expansions are similar in form to those obtained here, but are not identical since the unknown boundary functions are expanded by means of trigonometric series rather than in terms of eigenfunctions $\mathbf{Z}_{n}$. Our approach shows that the series expansions can be directly obtained by a generalized Fourier analysis without appeal to transform methods.

${ }^{1}$ See footnote after Eq. (3.6).

2See footnote on Page 71 of [6]. 


\section{REFERENCES}

1. M. W. Johnson, Jr., The elastic strip under end loading, Summary report, U. S. Army Mathematics Research Center, University of Wisconsin, Madison, to appear

2. E. L. Reiss, $A$ theory for the small rotationally symmetric deformations of cylindrical shells, Comm. Pure Appl. Math. 13, 531-550 (1960)

3. E. L. Reiss, On the theory of cylindrical shells, Quart. J. Mech. Appl. Math. 15, 325-338 (1962)

4. K. O. Friedrichs and R. F. Dressler, A boundary-layer theory for elastic plates, Comm. Pure Appl. Math. 14, 1-33 (1961)

5. R. C. T. Smith, The bending of a semi-infinite strip, Australian J. Sci. Res. 5, 227-237 (1952)

6. G. Horvay, Biharmonic eigenvalue problem of the semi-infinite strip, Quart. Appl. Math. 15, 65-81 (1957)

7. G. Horvay and J. S. Born, Some mixed boundary-value problems of the semi-infinite strip, J. Appl. Mech. 24, 261-268 (1957).

8. R. W. Little, Ph.D. dissertation, University of Wisconsin, 1962.

9. E. A. Coddington and N. Levinson, Theory of ordinary differential equations, McGraw-Hill, New York, 1955

10. A. P. Hillman and H. E. Salzer, Roots of $\sin z=z$, Phil. Mag. 34, 575 (1943)

11. C. I. Robbins and R. C. T. Smith, $A$ table of roots of $\sin z=-z$, Phil. Mag. 39, 1004-1005 (1948)

12. J. P. Benthem, A Laplace transform method for the solution of semi-infinite and finite strip problems in stress analysis, Quart. J. Mech. Appl. Math. 16, 413-429 (1963) 